\title{
Klinisch reiner Fall von spastischer Spinalparalyse (Erb) als Unfallfolge.
}

\author{
Von \\ Prof. Dr. A. v. Sarbó, Budapest.
}

(Mit 1 Abbildung.)

Die Publikation des nachstebenden Falles ist in mebrfacher Hinsicht motiviert. Vor allem zeigte derselbe in voller klinischer Reinheit das Bild der spastischen Spinalparalyse, wie dasselbe von Erb and Charcot entworfen wurde, und andererseits konnte als Ursache der Erksankung mehrfache Überanstrengung eruiert werden, ausserdem liefert dieser Fall den selten vorhandenen Beweis, dass in der Pathogenese organischer Erkrankungen Überanstrengungen eine wesentliche Rolle spielen kënnen.

Den Impuls zur Mitteilung dieses Falles gab mir ein in vor nicht langer Zeit erschienener Aufsatz Erbs, in welchem er eine spinale Muskelatrophie beschreibt, die er als Folge einer Überanstrengung ansieht und zur Erklärung die Edingersche Aufbrauchtheorie verwendet.

Erb weist in besagtem Aufsatz darauf hin, dass bis jetzt vornehmlich solche von durch Überanstrengung hervorgerufenen Erkrankungsfälle publiziert worden sind, in denen das spino-muskuläre Neuron als angegriffen bezeichnet werden konnte. "Wie verhält es sich aber", fragt Erb, „mit dem cortiko-spinalon Neuron, mit der Py-Bahn? Diese ist ja zweifellos garnicht selten miterkrankt! Viele Fragen tauchen da auf: Ist dieselbe primär erkrankt und durch das gleiche Geschehen? Ist ihre Störung vielleicht ganz besonders von dem psychischen Tranma abhängig, oder tritt sie vielleicht erst infolge der Erkrankung in den grauen Vordersäulen rückläufig auf? Wir wissen das alles noch nicht, und die Kasuistik, die darüber Aufschluss geben könnte, ist noch zu schaffen." -

Als einen bescheidenen Anfang in dieser Kasuistik erlaube ich mir meinen Fall zu publizieren.

Johann Sz., 43 Jahre alt, verheiratet, Kutscher. -

Negiert Lues gehabt zu haben. - Zwei lebende, gesunde Kinder. Seine Frau hat nie abortiert. - Kein Abusus spirituorum. - 
Ich sah Patienten pro consilio im November 1909 das erste Mal; seine einzige Klage bezog sich darauf, dass seine Füsse den Dienst versagen. Die Entwicklung seines Leidens liess sich folgendermassen konstruieren. Beginn des Leidens im Jahre 1902, April. - Die an Wagen gespannten zwei Fohlen wurden wild und rasten einige Kilometer davon, bis sie zwischen zwei elektrische Wagen gerieten, wo auch ein Lastwagen bemerkbar wurde, - Patient schrie aus Leibeskräften, konnte aber den Zusammenstoss nicht mehr aufhalten. Die Fohlen fielen um, dem Kutscher ist nichts passiert. Die Zügel waren vor Anstrengung in seine Hände eingeschwollen, er konnte kaum gehen.

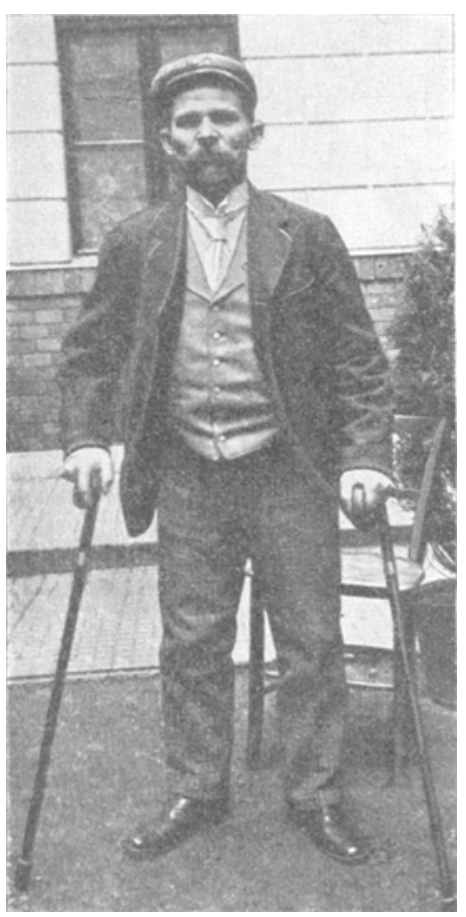
Er erinnert sich, während der ganzen etwa viertelstitndigen Dauer des Dahinrasens seine Füsse aus voller Kraft angestemmt zu haben. Vom nächsten Tag an bemerkte er ein Zittern in den Füissen, suchte einen Arzt auf, der ihn in den folgenden Jahren behandelte. Frr bekam Bäder und spirituöse Einreibungen für die Füsse. Er ging die ganze Zeit seiner Arbeit nach und konnte als Kutscher zur vollsten Zufriedenheit seiner Herrschaft den Dienst versehen.

Im Oktober 1907 zerbrach die Querstange seines Wagens und schlug an die Schenkel eines Pferdes, worauf beide wild wurden und davon rasten; mit äusserster Anstrengung konnte er die Tiere bändigen.

Nach diesem zweiten Unfalle verschlechterte sich der Zustand merklich, die Gehfähigkeit nahm ab, trotzdem versah er seinen Dienst weiter, als im Februar 1909 ein neuerlicher Unfall derselben Art ihn gehunfähig machte. Die Pferde scheuten vor einem Automobil und rasten ungefähr $7-8$ Kilometer lang, bis es ihm mit äusserster Kraftanwendung gelang, sie zum Stehen zu bringen.

Nach diesem letzten Unfalle verschlechterten sich die Füsse, am Morgen nach dem Unfalle konnte er die Füsse nur mehr schleppen, trotzdem versah er noch einige Tage den Dienst, allerdings mit äusserster Willensanstrengung, Anfäng März war er dann fast vollständig gelähmt.

Status praesens. Mittelweite, gleiche, gut reagierende Pupillen. Cerebrale Nerven funktionieren tadellos. - Obere Extremitäten ohne Befund. - Knochensystem normal. - Beide untere Extremitäten sind fast total gelähmt. Gehen kann er nur mit äusserster Anstrengung, er schiebt mehr seine Beine, dieselben sind im Knie etwas gebeugt, der Oberkörper nach vorne sich senkend, steif (siehe Abbildung). Stark entwickelte Muskulatur. Hochgradige Rigidität. Spastische Kniereflexe. Fussklonus. Beiderseitig Babinski. 
Kremaster- und Bauchreflexe normal.

Nirgends sensible Störungen. -

Nirgends Muskelatrophien. -

Beim Urinieren muss er drängen.

Stuhl hat er nur einmal in der Woche.

Schmerzen hat er nie gehabt auch jetzt klagt er nicht über solche. Wassermannsche Seroreaktion negativ.

Energische Schmierkur und Jod ohne Erfolg angewendet, nach einem Jahre ist der Status unverändert. -

Resumé. Ein bis dahin gesunder 36 jähriger Kutscher bemerkt eine Schwäche und Zittern in den Füssen nach einem Untalle, welcher darin bestand, dass seine Pferde scheuten, viertelstundelang dahinrasten, ohne dass er sie bändigen konnte, bis er endlich zwischen zwei elekrische Wagen geriet, wo er einen entgegenfahrenden Lastwagen erblickend aus Leibeskräften schreit, die Pferde rennen an den Wagen an und bleiben endlich stehen. Von diesem Moment an bemerkt er; dass seine Füsse schlottern und schwach sind und es auch weiterhin bleiben. Trotzdem setzt er seine Besehäftigung fort. Nach 5 Jahren scheuen die Pferde neuerdings, rasen 5-6 Kilometer weit, bis es ihm durch äusserste Kraftanwendung gelingt, sie zu bändigen. Nach diesem zweiten Unfall versehlechtert sich der Zustand der Füsse, er kạn nur mühsam gehen, trotzdem geht er seiner Beschäftigung nach, bis dann ein dritter, dem ersten gleicher Unfall nach zwei Jahren ihn ganz herunterbringt. Als ich ihn nach diesem letzten Unfall untersuche, finde ich das klassische Bild der spastisehen Spinalparalyse.

Trotz negativer Luesanamnese, trotz negativen Wassermanns verordnete ich eine energische antiluetische Kur, jedoch vollkommen ohne Erfolg.

Die klinische Diagnose ist kaum zu bezweifeln. Alle jene übrigen Erkrankungen, welche mit spastiseher Paraplegie einhergehen, als amyotrophische Lateralsklerose, Sclerosis multiplex, Syringomyelie, Tumor medullae spinalis, sind beim Fehlen irgendeines für dieselben charakteristischen Symptoms vollkommen auszuschliessen.

Als ich den Kranken untersuchte, währte die Erkrankung schon seit 8 Jahren. Im Laufe dieser Zeit müssten sich auch andere Symptome gezeigt haben, wenn es sich um eine der oben genannten Krankheiten handeln sollte. An eine funktionelle Erkrankung ist bei der Anwesenheit der charakteristischen organischen Symptome nicht zu denken, es fehlten übrigens auch irgendwelche hystero-neurasthenische Symptome.

Der anatomishe Hintergrund des beobachteten spastisehen Symptomenkomplexes kann nur im kortiko-spinalen Neuron gesucht werden; irgendein Symptom, welehes auf die Erkrankung der peripheren Ner- 
ven oder auf irgendeine andere Stelle des Zentralnervensystems hingedeutet hätte, war nicht eruierbar. Das erschwerte Urinieren sowie die hochgradige Stuhlverstopfung lassen sich mit der Annahme der Pyramidenläsion aber gut in Einklang bringen, wie dies übrigens auch schon Eduard Müller in einem ähnlichen Fall des näheren ausführte.

Wir erblicken in diesen Störungen der Miktion und Defäkation ein den Muskelspasmen in den unteren Extremitäten analoges spastisches Symptom.

Klinisch entspricht unser Fall ganz den Anforderungèn, die Erb, dem wir die Aufstellung des Symptomenbildes der Paralysis spinalis spastica verdanken, fordert. Dasselbe besteht aus dem Symptomenquartett, wie sich Erb ausdrückt: Parese, Hypertonie, spastische Sehnenreflexe, Babinskis Zeichen. -

Bekanntlich hatte Erb dieses Krankheitssyndrom schon im Jahre 1872 aufgestellt, vorerst nur auf klinischer Grundlage, bald folgten Charcots Beobachtungen. Letzterem verdanken wir die Bezeichnung Tabes dorsal spasmodique. Aber erst im Jahre 1902 konnte Erb den pathologisch-anatomischen Nachweis dafür liefern, dass seine Hypothese, wonach die Ursache der spinalen spastischen Paralyse in der isolierten Erkrankung der Pyramidenbahn zu suchen sei, richtig war.

In seinem im West London Hospital gehaltenem Vortrage konnte Erb schon über 11 obduzierte Fälle referieren, von denen in 6 Fällen das Postulat der reinen Pyramidenerkrankung erfüllt gewesen ist; in 4 Fällen waren ausserdem leichte Degenerationen in der Kleinhirnbahn, und in einem Fall waren die Kleinhirnbahn und die Gollschen Stränge leicht lädiert.

Erb sagt hierüber wörtlich Folgendes: „In allen 11 Fällen war die Degeneration der Pyramiden das weitaus Überwiegende; sie erschien intensiver, mit allen Charakteren einer primären, von unten bis gegen die Oblongata reichenden, allmählich abnehmenden Sklerose; die neben diesen noch gefundenen Veränderungen der Kleinhirnseitenstrangbahn und der Goll schen. Stränge erscheinen untergeordnet und sind, weil klinisch gänzlich symptomlos, wohl ausser Betracht zu lassen."

Erbs Befunde wurden durch spätere Beobachter bestätigt.

Strümpell, dem wir wichtige Aufschlüsse über die Systemerkrankungen verdanken, bespricht im Jahre 1904 die primäre Seitenstrangsklerose und bringt den Sektionsbericht seines berühmten Falles Polster, den er 15 Jahre hindurch beobachten konnte. Auf Grund dieses sowie noch zweier anderer obduzierter Fälle hält er das Vorkommen einer primären systematischen Degeneration der Pyramidenseitenstrangbahn für absolut gesichert. 
Dejerine und Sottas, Schüle, E. Müller, Kattwinkel, Newmark, Spiller u. a. haben dann weitere bestätigende Fälle mit Obduktionsbefund mitgeteilt.

Die viel umstrittene Frage der primären systematischen Rückenmarkserkrankung fand in diesen Publikationen zu gunsten der Existenz einer solchen eine starke Stütze.

Von der Erbschen ursprünglichen Auffassung über die isolierte systematische Py-Degeneration weicht Dejerine in der Nuance ab, dass er auf Grund seines Befundes nur die Möglichkeit einer kombinierten Systemerkrankung zugibt, da er auch in seinem Fall neben stark ausgesprochener Py-Degeneration minimale Degenerationen im cervikalen Anteil der Gollschen Stränge vorfand.

Trotz der Seltenheit diesbezüglicher Fälle existiert nach Dejerine eine primäre spastische Spinalparalyse und ist dieselbe nur klinisch rein, der pathologisch-anatomische Hintergrund ist selbst in den reinsten Fällen eine kombinierte Sklerose.

Die Frage wurde noch dadurch kompliziert, dass immer mehr Fälle publiziert worden sind, in denen das familiäre Auftreten dieses Leidens beobachtet wurde. Schon Strümpell weist auf diesen endogenen Faktor hin, die Beobachtungen von Sidney Kuh, Voss u. a stützten diese Ansicht. Fs war nicht zu verwundern, dass diejenigen Forscher, die mit den heredodegenerativen Krankheiten sich befassten, auch die spinale Paralyse unter diese Erkrankungen einreihten, um so mehr, als gewichtige Stimmen (z. B. Leyden u. a.) gegen die Existenz einer primären systematischen Erkrankung laut wurden.

So hat sich dann die neueste Auffassung über die Pathogenese dieser Erkrankung herangebildet, als deren hauptsächlichster Vertreter Jendrássik zu betrachten ist.

Jendrássik leugnet die nosologische Sonderstellung der spastischen Spinalparalyse und erblickt in ihr nur ein Glied der hereditären Krankheiten. Er selbst hat einen Fall publiziert, in welchen die Spinalparalyse als Folge einer sekundären Degeneration nach einer Myelitis aufgefasst werden konnte.

Am radikalsten verfährt Lewandowsky, der in einer Fussnote des Jendrássikschen Aufsatzes nach Abfertigung der Existenz echter Systemerkrankungen zugibt, dass es vielleicht doch sehr vereinzelte Fälle geben kann, wo zu den familiär-hereditären zu rechnende, aber anscheinend freistehende primäre Degeneration vorkomme. Als einen solchen Fall erscheint ihm der von E. Müller beschriebene.

Aus dem Vorgetragenen ersehen wir, dass die Frage, ob eine primäre systematische Degeneration existiere, noch in Schwebe ist. Meiner Ansicht nach entstanden diese einander widersprechenden 
Meinungen daher, dass wir über die ätiologischen Faktoren noch heute im Unklaren sind. Diejenigen Forscher, die entgegen der übertriebenen nosologischen Zersplitterungen eine allgemeinere umfassendere Betrachtungsweise zur Geltung bringen wollen, indem sie in der Heredodegeneration den Schlüssel der Erkrankung erblicken wollen, betonen meines Erachtens nach zu sehr dieses eine ätiologische Moment; ist es doch sicher, dass es ausser diesem familiär-hereditären Faktor noch andere Bedingungen der Erkrankung gibt. Es steht zu befürchten, dass die übermässige Betonung des hereditären Faktors zu der Ansicht führt, dass wir nun auch die Ursache des Leidens kennen und damit die Erforschung der übrigen mitwirkenden Faktoren vernachlässigen. Wir müssen Verworn in der Forderung beipflichten, bei den naturwissenschaftlichen Forschungen mit der alten. Ansicht zu brechen, wonach man ein naturwissenschaftliches Phänomen bei der Auffindung "der Ursache" schon erklärt und dabei vergisst, dass es kein Phänomen gibt, fwelches nur eine Ursache hätte, sondern ein jedes Phänomen ist durch zahlreiche Faktoren bedingt.

Es ist nötig, dass der mystische Kausalismus den Platz dem wissenschaftlichen Konditionalismus räumt - sagt mit Recht Verworn.

Im mitgeteilten Fall dürfen wir uns auch nicht damit begnügen, als Ursache der Erkrankung einfach die endogene Minderwertigkeit der Pyramidenbahn vorauszusetzen, sondern es ist nötig zu betonen, dass aller Wahrscheinlichkeit nach die mehrmaligen Überanstrengungen als dasjenige Moment zu bezeichnen sind, welches im Chemismus der Pyramidenbahn jeneGleichgewichtsstörung hervorgerufen hat, als deren Folge die Degeneration der Pyramidenbahn eintrat. Auch die weitere Frage erheischt Antwort, welche Stelle der Pyramidenbahn - die Zellen oder die Fasern - als entartet zu betrachten ist. Oder handelt es sich gar bei intakter Pyramidenbahn um Störungen der den Pyramidenzellen superponierten sogen. intrakortikalen Neuronen - wie wir sie aus den Schilderungen Spielmeyers kennen?

Spielmeyer machte uns mit Fällen bekannt, in denen bei intakter Pyramidenbahn der spastische Symptomenkomplex zu beobachten war und sich als anatomische Läsion Atrophie der Gehirnoberfläche vorfand. Der erste Fall Spielmeyers bezog sich auf einen epileptischen Kranken, bei welchem nach einem Status epilepticus eine spastische Hemiplegie auftrat. Die mikroskopische Untersuchung erwies, dass sowohl die Pyramidenfasern als auch die grossen Pyramidenzellen intakt waren. Hingegen ergab sich eine der Hemiplegie kontralaterale Atrophie der Hirnrinde. Mit dieser letzteren musste die spastische Hemiplegie in Verbindung gebracht werden, d. i. es muss 
vorausgesetzt werden, dass es den grossen Pyramidenzellen übergeordnete Neuronen gibt und die Lädierung dieser intrakortikalen Neuronen dasselbe klinische Bild hervorruft als die Läsion der Py-bahn.

Spielmeyer folgert aus diesem Fall, dass auch sonst manche von den spastischen Lähmungen, die man bei chronischen Rindenerkrankungen beobachtet und die man nach den bisherigen Erfahrungen auf eine Läsion der Py-bahn zurückzuführen pflegt, auf solchen intrakortikalen Veränderungen beruhen könnten. Er denkt dabei an manche epileptischen Erkrankungen mit Halbseitenerscheinungen und an Fälle von sogen. atypischer seniler Demenz mit spastischen Lähmungen.

Er erwähnt auch einen diesbezüglichen Fall, in welchem es sich um eine durch drei Jahre fortschreitende spastische Paraplegie handelte bei einer 53jährigen Frau, die psychisch das Bild einer präsenilen Demenz aufwies. Der Rindenprozess war zwar ein anderer als der im erstem Falle, jedoch war die Übereinstimmung darin, dass auch in diesem zweiten Fall die Pyramidenzellen und Py-bahn intakt waren, woraus Spielmeyer den Schluss zieht, dass der Py-bahn Neurone übergeordnet sind, deren Läsion eine gleichartige, grobe, motorische Störnng mit Spasmen, Kontrakturen und dem Babinskischen Phänomen bewirken kann, wie sie sonst die Läsion der Py-bahn verursacht.

Zurückkommend auf unseren Fall, können wir das eventuelle Vorhandensein solch einer intrakortikalen Läsion (Spielmeyer) deshalb mit aller Wahrscheinlichkeit ausschliessen, weil sowohl irgendwelche auf kortikale Beteiligung hinweisende psychische als auch epileptische Symptome rollkommen fehlten. Wir können daher mit grosser Wahrscheinlichkeit behanpten, dass in unserem Fall die Symptome auf eine Läsion der Py-bahn zu beziehen sind. Entscheiden können wir die Frage nicht, ob die Py-zellen oder nur die Fasern oder beide zugleich Sitz der Läsion sind. Rechnen wir unseren Fall zu den ähnlichen von Heredodegeneration und supponieren wir in demselben denselben Degenerationsprozess, der bei sezierten Fällen dieser Art bis jetzt vorgefunden worden ist, so müssen wir aus diesen Erfahrungen schliessen, dass auch in unserem Fall es sich um eine Degeneration der Py-fasern im Rïckenmark handeln muss, welche in der Mednlla oblongata aufhört. Wir haben aber schon oben auseinandergesetzt, dass wir uns mit dieser einseitigen Auffassung des Symptomenkomplexes als Heredodegeneration nicht begnügen dürfen, sondern trachten müssen, auf die Frage, welche Stelle der Py-bahn wir als erkrankt betrachten können, aus dem klinischen AneinanderDeutsche Zeitschrift !. Nerverheilkunde. Bd. 46 . 
reihen der Symptome sowie aus dem ganzen Verlauf und der Entwicklung der Krankheit die Antwort, wenn auch nur bypothetisch, zu geben.

Bevor wir diese Frage beantworten, müssen wir dariiber ins Reine kommen, ob denn überhaupt im Einklang mit unseren heutigen pathogenetischen Begriffen die Annahme, dass Überanstrengung eine organische Läsion erzeugen könne, möglich sei.

Diese Frage wurde schon von Erb berüht und im bejahenden Sinne für das Entstehen der amyotrophischen Spinalerkrankungen beantwortet. Als Erklärung der Wirksamkeit dieses Faktors zieht Erb die Edingersche bekannte Aufbrauchtheorie heran.

Es ist durch mehrfache Beobachtungen erwiesen, dass Überanstrengungen solche klinische Symptome vorübergehend erzengen können, die wir gemeiniglich als organisch bedingt aufzufassen pflegen.

Ich weise diesbezüglich auf Auerbachs Untersuchungen hin, der bei Radfahrern als Ermüdungssymptom vorübergehenden Patellarreflexverlust finden konnte. Unter seinen Beobachtungen finden wir drei Fälle, in denen Reflexsteigerung eingetreten war.

Knapp und Thomas (zitiert nach Oeconomaki) fanden bei Rennern sehr oft Reflexsteigerungen und fassen dieselben als Folge der psychischen Erregung auf.

Oeconomaki hat bei der Untersuchung an den Marathonläufern nicht nur den Patellar-, sondern anch den Achillessehnenreflex untersucht.

Er fand in sechs Fällen Steigerung des Kniescheibenreflexes, in acht Fällen dasselbe Symptom von seiten des Achillesphänomens. In vier Fällen Patellarareflexie und in einem Fall Achillesareflexie.

Wir erinnern an die experimentellen Untersuchungen von Edinger and Helbing. Diese Autoren haben bei am Schwanze aufgehängten und zappelnden Ratten im Rückenmark Faserausfall in den Hintersträngen nachweisen können. Der Faserausfall war noch grösser bei solchen Tieren, die ansserdem noch mit Pyridin vergiftet worden sind.

Sternberg erwähnt in seiner bekannten Monographie über die Sehnenreflexe, dass Kummer und Sorge reflexsteigernd wirken; er zitiert die Untersuchungsergebnisse Leonhards, der seinen Patellarreflex unter dem Einflusse intensiver Gemütsbewegungen gesteigert fand.

„Jede körperliche, jede geistige Anstrengung“, sagt Sternberg, „steigert, sobald sie zur allgemeinen Ermüdung führt, sobald sich das Gefühl der Abspannung und Ermattung einstellt, die Sehnenreflexe. Es kommen in Betracht: längere Märsche, Bergtouren, angestrengtes 
Reiten...., angestrengte körperliche Arbeit im engeren Sinne, geistige Arbeit."

Nach Sternbergs Ansicht wäre diese Reflexsteigerung dadureh zu erklären, dass die Ermüdung auf das Gehirn einwirkt und dass dadurch die cerebrale Hemmung ausbleibt.

Diese Steigerung nach Ermüdung, sagt Sternberg, ist wiederholt entdeckt worden. „Die erste Erwähnung des steigernden Einflusses lang danernder Muskelarbeit findet sich bei Brissaud. Marimian bespricht in seiner sehr sorgfältigen Arbeit Steigerung nach Ermüdung. Schuster beschreibt Steigerung nach körperlichen Anstrengungen, Nachtwachen usw. Erlenmeyer beobachtete die gleiche Erscheinung nach Bergtouren und Reiten ...."

Es ist eine bekannte klinische Erfahrung, dass die stärkste Reflexsteigerung bei den Läsionen der Py-bahn zu beobachten ist; spastische Sehnenreflexe, Babinskis Zeichen, Hypertonie bilden das klinische Bild dieser Reflexsteigerung.

Als Erklärung dieses Zusammenhanges wird heute allgemein angenommen, dass die spastischen Symptome bei Läsionen der Pybahn dadurch zustande kommen, dass die cerebrale Hemmung bei der Degeneration der Py-bahn wegfällt.

Die Frage wurde in diesem Sinne durch die Beobachtungen an Säuglingen, sowie durch die Fälle der Agenesien der Py-bahn klargestellt. Die Beobachtungen an Sänglingen haben gezeigt, dass bei ihnea eine sehr hohe Reflexsteigerung de norma ist, die so weit gehen kann, dass beim Beklopfen der einen Patellarsehne beide vierköpfige Muskeln in lebhafte Zuckung geraten. In den Fällen von Agenesie der Pybahn finden wir sehr oft den spastischen Symptomenkomplex. Die Säuglingsreflexerhöhung findet ihre Erklärung darin, dass die Py-bahn. in noch nicht funktionsfertigem Zustande ist, da die Myelinisation der Py-seitenstränge erst im neunten Monat beendigt ist. In den Fällen der Agenesie der Py-bahn ist eben dieses Fehlen derselben als Ursache der spastischen Zustände zu betrachten. In den angeführten Beispielen bekommen wir denselben Symptomenkomplex der Reflexsteigerung zu sehen als bei Degeneration dex Py-bahn - das Gemeinschaftiche in allen diesen Fällen ist darin zu erblicken, dass der cerebrale Impuls aus den Py-zellen nicht fortgeleitet werden kann, so dass wir mit Wahrseheinlichkeit folgern dürfen, dass der spastische Symptomenkomplex bei Läsionen der Py-bahn auf das Fehlen der cerebralen Hemmung zurückzuführen ist.

Kehren wir nach diesen langen Erörterungen zu unserem Fall zurück, so können wir feststellen, dass der in demselben zu beobachtende Symptomenkomplex unter solchen Umständen entstanden 
ist, die mit Reflexsteigerungen einhergehen, wofür wir im Vorhergehenden den Nachweis lieferten.

Aus den zitierten Beobachtungen folgt, dass die Ermüdung des Zentralnervensystems sehr oft mit Reflexsteigerung einhergeht. Betrachten wir unter diesem Gesichtspunkte unseren Fall, so können wir konstatieren, dass eine maximale Inanspruchnahme des Zentralnervensystems zweifellos stattfand und es folgerichtig ist, wenn wir die beobachtete Reflexsteigerung als Folge der Ersehöpfung damit in Verbindung bringen. Wir vergessen dabei nicht, dass es sich in dem angeführten Beispiel nur um passagere Reflexsteigerungen handelte und auch die Reflexsteigerung nicht die Höhe der Spastizität erlangte wie in unserem Falle -, trotzdem glauben wir ein gleiches Geschehen in beiden Fällen voraussetzen zu dürfen und in der Hochgradigkeit der Reflexsteigerung in unserem Falle nur eine quantitative Differenz gegenüber den Reflexsteigerungen bei Ermüdungen zu erblicken.

Es unterliegt keinem Zweifel, dass der Kraftaufwand, den unser Patient bei dem ersten Unfall aufbringen musste, sein Zentralnervensystem maximal in Anspruch nahm.

Vergegenwärtigen wir uns seine Lage: Er musste gescheute Fohlen zurückhalten, indem er sich mit äusserster Kraftentfaltung mit den Füssen dem Bocke entgegenstemmend, mif den Armen krampfhaft die Zügel anspannte. Zu alledem kam noch der psychische Shock, der noch gesteigert wurde, als er zwischen zwei elektrische Wagen kommend, einen anderen ihm entgegenfahrenden Wagen erblickte, was ihn zu wildem Warnungsrufen veranlasste. Wir behaupten wohl mit Recht, dass er seiner ganzen Kraftentfaltung benötigte, um Herr der Situation zu bleiben. Bei dieser Kraftentfaltung muss sich das ganze Zentralnervensystem beteiligt haben; am stärksten war selbstredend die motorische Bahn in Anspruch genommen. Es fragt sich nun, woher es kommt, dass in diesem Falle nicht die Zellen des Vorderhorns des Rückenmarks erkrankten, sondern die Py-bahn. Nach den Erbschen Fällen wäre allerdings eine spinale Amyotrophie als Folgekrankheit zu erwarten gewesen. Dass nicht diese, sondern die Py-bahn erkrankte, wollen wir dadurch erklärt wissen, dass von beiden Bahnen doch die kortikospinale Bahn es war, deren Anstrengung eine grössere gewesen ist, als die der spinomuskulären. Die kortikospinale Bahn hatte in der Situation, in welcher sich unser Patient befand, die Aufgabe, die richtige Koordination der angespannten Muskulatur zu überwachen und die Muskeln in Spannung zu halten. Ich habe an mir selbst die Erfahrung wiederholt machen können, dass ich im offenen Wagen fahrend - beim Anblick eines 
zu gewärtigenden Zusammenstosses mit einem anderen Fuhrwerk oder beim Scheuen der Pferde :- nnwillkürlich mit grosser Energie mit den Füssen mich "entgegenstemmte -; diese Bewegung ist sicher kortikospinalen Ursprunges. In übertriebener Weise hat unser Patient dieselbe Bewegung dauernd ausgeführt und zu all dem noch den psychischen Shock erleiden müssen.

Ich will mich nicht in weitere Erörterungen einlassen und begnüge mich mit der vorgetragenen plausiblen Erklärungsweise, unterlasse die Aufstellung weiterer Möglichkeiten, verzichte darauf zu erörtern, ob es nicht richtiger wäre, daran zu denken, dass in unserem Falle die Py-bahn endogen bedingt schwächer veranlagt war als das spinomuskuläre Neuron - und darin der Grund der Erkrankung des kortikospinalen Neurons zu suchen sei!

Die Frage muss aber noch besprochen werden, ob solche funktionelle Überanstrengung imstande ist, materielle Läsionen hervorzubringen - denn das eine müssen wir als sicher annehmen, dass bei keinem der Unfälle unseres Patienten ein Trauma stattgefunden hat.

Als Antwort auf diese Frage berufe ich mich so wie Erb auf die Aufbrauchtheorie Edingers, wonach die Funktion allein imstande ist, wenn keine Restitution eintritt, zur Degeneration von Nervenbahnen zu führen.

Verw orn s grundlegende Experimente an strychninisierten Fröschen werfen ein interessantes licht auf die in den Nerverzellen sich abspielenden Prozesse.

„Unter normalen Verhältnissen, bei ungestörter ruhiger Tätigkeit halten sich die beiden Phasen. des Stoffwechsels in den Neuronen das Gleichgewicht. Es wird ebensoviel lebendige Substanz gebildet, wie zerfällt. Der Blutstrom sorgt für Zufuhr des Assimilationsmaterials und für Abfuhr der Dissimilationsprodukte. Anders wird das Verbältnis bei angestrengter Tätigkeit. Das Stoffwechselgleichgewicht wird gestört, der Zerfall wird grösser als die Neubildung. Die Assimilation kann nicht gleichen Schritt halten mit der Dissimilation. ..... Je länger die Ganglienzelle arbeitet, um so mehr entwickeln sich Lähmungserscheinungen. . . . Diese Lähmungserscheinungen beruhen auf zwei verschiedenartigen Ursachen, die beide in demselben Sinne wirken. Die eine ist die Anhäufung von lähmend wirkenden Zersetzungsprodukten der lebendigen Substanz, wie Kohlensäure, die andere der Mangel an Ersatzmaterial und zwar zunächst allein an Sauerstoff."

Bei einer sol.ch hochgradigen Überanstrengung wie in unserem Falle bei dem ersten Unfall ist es erlaubt vorauszusetzen, dass der Chemismus der Ganglienzellen durch die Anhäufung der Zersetzungs- 
produkte derartig gestört werden konnte, dass daraus eine organische Veränderung der Zellen resultierte. Da die Py-bahn, wie wir dies oben auseinandersetzten, im gegebenen Falle stärker in Anspruch genommen ward, ist es erklärlich, dass sie es war, welche die irreparablen Veränderungen erfuhr, während die spinalen motorischen Ganglienzellen sich erholten. Die erste Überanstrengung hat nur zu einer Schwächung der Py-bahn geführt; sofort nach dem Umfalle beginnen die Füsse zu zittern und bleiben gegen früher geschwächt - der Patient suchte forf and fort ärztliche Hilfe dagegen. Trotz dieser Schwächung der Füsse konnte er seinen Beruf als Kutscher fortsetzen, es trat auch keine weitere Verschlechterung ein, was wir darauf zurückzuführen geneigt sind, dass er in seiner sitzenden Beschäftigung seine Füsse nicht weiter anstrengte. So konnte er zur vollsten Zufriedenheit seines Arbeitsgebers seinen Beruf noch fünf Jahre lang erfüllen, da kam die zweite der ersten ähnliche Überanstrengung. - Der Zustand verschlechterte sich zusehends, trotzdem arbeitete er, wenn auch mühsam, weiter, um nach weiteren zweieinhalb Jahren den dritten ähnlichen Unfall zu erleiden, und die von den vorhergehenden C̈beranstrengungen abgeschwächte kortikospinale Bahn versagt nun gänzlich den Dienst, und ich finde das klassische Bild der spastischen Spinalparalyse, aber aller Wahrscheinlichkeit nach totales Zugrundegehen der Py-bahn.

Überblicke ich die mir zugänglichen Fälle von spastischer Spinalparalyse in Bezug auf die Ätiologie, so finde ich in einigen Fällen Hinweise auf Überanstrengungen. So z. B. teilt Friedmann einen Fall von einer 49 jährigen Patientin mit, die mit 14 Jahren an einer ausgesprochenen und reinen spastischen Lähmung der Beine litt, welche ziemlich rasch nach einem. anstrengenden Spaziergang an der Nordsee aufgetreten sein soll; zunächst bestand nur leichte Ermüdbarkeit, erst nach einigen Jahren Unfähigkeit zu gehen unter ganz allmählicher Steigerung der Parese.

Nach Kuh kommen neben der Syphilis als auslösende Momente nur noch den Erkältungen und ebenfalls den Traumen und Überanstrengungen eine wesentliche Bedeutung zu.

Auch psychische Traumata sollen ab und zu eine Rolle gespielt haben. So in zwei Fällen Schüles. * Fall 1 ein 42 jähriger Mann, bei dem ein halbes Jahr nach einem heftigen Schreck sich die ersten Symptome der Erkrankung zeigten. Patient war nach dem Schrecken einige Stunden wie gelähmt, „auch früher schon hatten sich bei heftigem Schreck solche Erscheinungen gezeigt, besonders im linken Bein“. Diese Bemerkung in der Anamnese scheint darauf hinzuweisen, dass in diesem Falle gehäufte psychische Traumata vorangegangen seien. 
Der zweite Fall Schüles bezieht sich auf einen 37 jährigen Mann, der seine Krankheit von einem sehr heftigen Schreck (Brandunglïck) herdatiert. „Am folgenden Tag schon empfand er ein Schwächegefuihl, besonders im linken Bein."

Das ist so ziemlich alles, was ich vorfinden konnte.

Zum Schluss will ich noch die Frage berühren, ob solche Überanstrengungen als Unfall zu bezeichnen sind oder nicht? Ich glaube, es kann keinem Zweifel unterliegen, dass diese Art von Überanstrengung den Kriterien des Unfallbegriffes entspricht, handelte es sich doch um die normalen Muskelleistungen weitaus übersteigende Anstrengungen.

In unserem Fall dachte der Patient gar nicht daran, Entschädigungsansprüche zu stellen, diesem Umstande war es wahrscheinlich zuzaschreiben, dass der Patient gar keine neurotischen Symptome aufwies. Ich. war derjenige, der seinen Zustand als Unfallfolge erfasste und die Einleitung der Entschädigung veranlasste. -

Sollten auch diese meine Auseinandersetzungen in Zakunft durch entscheidendere Beobachtungen über den Haufen geworfen werden, so steht doch so viel fest, dass damit vom Standpunkte der Unfallversicherung in ähnlichen Fällen die Überanstrengung als wichtiges anslösendes Moment anerkannt werden muss, da unsere Unwissenheit nicht zum Nachteil des zum Schaden Gekommenen ausgenutzt werden darf. --

\section{Literatur.}

1) Erb, Zur Lehre von den Unfallserkrankungen des Rückenmarks usw. Deutsche Zeitschr. f. Nervenheilkde. Bd. $45,1$.

2) Derselbe, Deutsche Zeitschr. f. Nervenheilkde. Bd. 23.

3) v. Strümpell, Deutsche Zeitschr, f. Nervenheilkde. Bd. 5 u. 27.

$4)$ Schüle, Deutsche Zeitschr. f. Nervenheilkde. Bd. 4.

万) Eduard Müller, Deutsche Zeitschr. f. Nervenheilkde. Bd. 29.

6) Kattwinkel, Deutsche Zeitschr. f. Nervenheilkde. Bd. 23.

7) Voss, Neurol. Zentralbl. 1909.

8) Friedmann, Deutsche Zeitschr. f. Nervenheilkde. Bd. 16.

9) Kuh, Deutsche Zeitschr. f. Nervenheilkde. Bd. 3.

10) Spielmeyer, Neurol. Zentralbl. 1909.

11) Edinger, Deutsche med. Wochenschr. 1905.

12) Verworn, Arch. f. Physiologie 1900. Supplementband.

13) Dejerine, Maladies de la moëlle.

14) Jendrássik, Lewandowsky in Lewaudowskys Hanabuch. Art. hereditäre Krankheiten. 